\title{
Nanomaterial Interactions with Biological Systems: Implications for Occupational Health
}

\author{
Ivo Iavicoli, ${ }^{1}$ Paul A. Schulte, ${ }^{2}$ and Sergio Iavicoli ${ }^{3}$ \\ ${ }^{1}$ Institute of Occupational Medicine, Catholic University of Sacred Heart, 00168 Rome, Italy \\ ${ }^{2}$ National Institute for Occupational Safety and Health, Centers for Disease Control and Prevention, Atlanta, GA 30333, USA \\ ${ }^{3}$ Department of Occupational Medicine, Italian Workers Compensation Authority (INAIL), 00198 Rome, Italy
}

Correspondence should be addressed to Ivo Iavicoli, iavicoli.ivo@rm.unicatt.it

Received 2 August 2012; Accepted 2 August 2012

Copyright (c) 2012 Ivo Iavicoli et al. This is an open access article distributed under the Creative Commons Attribution License, which permits unrestricted use, distribution, and reproduction in any medium, provided the original work is properly cited.

Nanotechnology is a system of innovative methods for controlling and manipulating matter at the near-atomic scale to produce engineered materials, structures, and devices. Engineered nanoparticles are generally considered to be a class or subset of nanomaterials with at least one dimension, that is, approximately 1 to 100 nanometers. The specific attractive properties of nanomaterials have led to their widespread application in many sectors, such as medicine, consumer products, energy, materials, and manufacturing. However, the limited but positive knowledge regarding engineered nanoparticle toxicological profiles, the potential greater biological reactivity due to the high-surface area-size ratio, and the increased likelihood of human exposure has raised several concerns about the potential adverse health effects for general and occupational exposed population. Additionally, there is a continuing interest in incidental nanoparticle exposure, not intentionally produced by anthropogenic sources, namely, processes involving combustion, welding, or diesel engines. The main scope of this special issue is to contribute original in vitro and in vivo research articles as well as review articles that seek to define the nanomaterial interactions with biological systems that could be relevant in understanding the implications of nanoparticle exposure for the workers' health. We have invited colleagues from worldwide who have been exploring their research in this field.

Concerning the in vitro studies, the paper by C. L. Ursini et al. focused on the effects of $\mathrm{OH}$-functionalized multiwalled carbon nanotubes (MWCNTs). The findings demonstrated their cytotoxicity, through reduction of cell viability and induction of apoptosis without cell membrane damage, and the genotoxicity, by direct DNA damage induction, suggesting that the MWCNTs enter the cell without damaging its membrane and directly interact with the nucleus. In another interesting in vitro study, A. Mishra et al. determined the fibrogenic potential of well-dispersed MWCNTs in human lung cell culture models and developed a novel platform for understanding the cellular mechanisms of MWCNT-induced lung fibrosis. Interestingly, the results indicated that the dispersion status of MWCNTs determines their fibrogenic activity which is consistent with in vivo findings.

Concerning the in vivo studies, J. M. Legramante et al. tested the hypothesis that single-wall carbon nanotubes (SWCNTs) pulmonary exposure acutely affect the autonomic cardiovascular regulation in conscious rats. Their results showed that in Wistar-Kyoto rats SWCNT pulmonary exposure can affect the cardiovascular autonomic regulation, thus contributing to cardiac and arrhythmic events. On the other hand, J. R. Roberts and colleagues assessed pulmonary toxicity and fate of silicon nanowires ( $\mathrm{Si} N W s$ ) in male Sprague-Dawley rats. Their findings demonstrated that Si NWs induced transient lung toxicity which may be associated with an early rapid particle clearance. Finally, the authors concluded that persistence of Si NWs over time related to dose or wire length may lead to increased collagen deposition in the lung. In contrast, ingestion exposure as illustrated by the study of Novak et al. of titanium dioxide nanoparticles $\left(\mathrm{TiO}_{2} \mathrm{NPs}\right)$ in a model invertebrate showed no internalization of consumed nanoparticles even when cytological markers strongly indicated an adverse effect.

Concerning $\mathrm{TiO}_{2} \mathrm{NPs}$, the review by I. Iavicoli et al. provides a comprehensive evaluation of the toxic effects induced by these nanoparticles in in vivo experiments. The main 
aim of this paper was to deeply understand the toxicological behaviour of $\mathrm{TiO}_{2}$ NPs and to predict potential human health effects.

Finally, the last two papers by E. Bergamaschi and M. Riediker et al. treated the applicability of biological monitoring to the assessment of exposure and the development of a control banding tool for nanomaterials, respectively. These authors proposed possible ways to assess and to manage the risk related to the nanomaterial exposure underlying the need for further future studies.

In conclusion, we hope this special issue will be informative to and ultimately contribute to the protection of workers.

Ivo Iavicoli

Paul A. Schulte

Sergio Iavicoli 

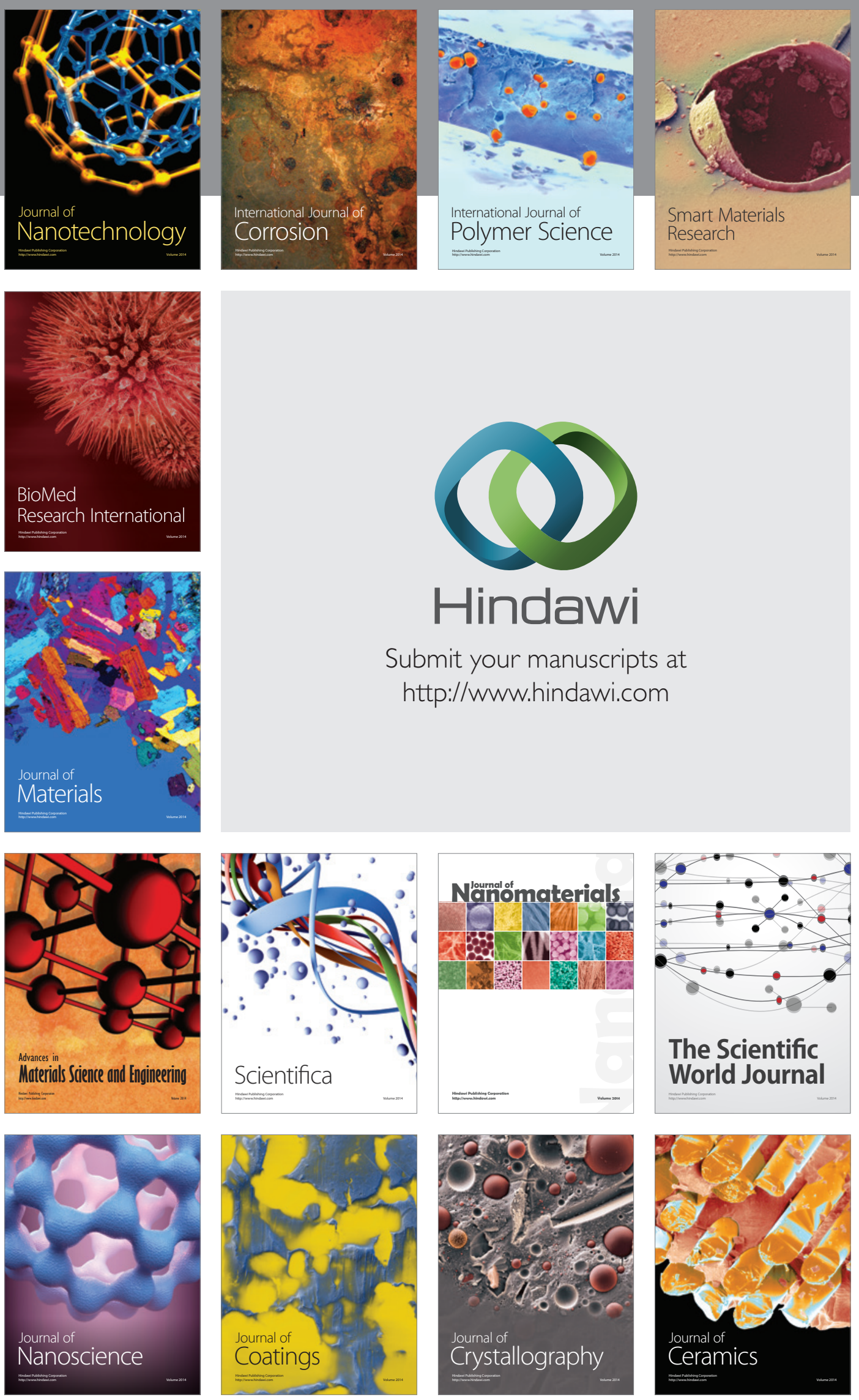

The Scientific World Journal

Submit your manuscripts at

http://www.hindawi.com

\section{World Journal}

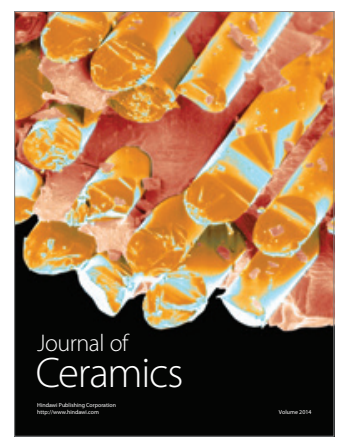

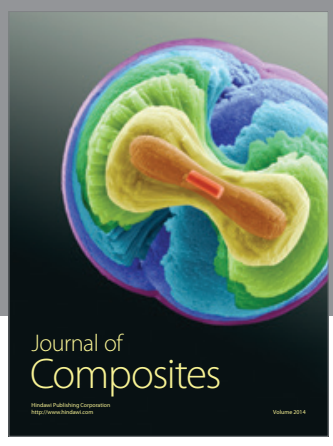
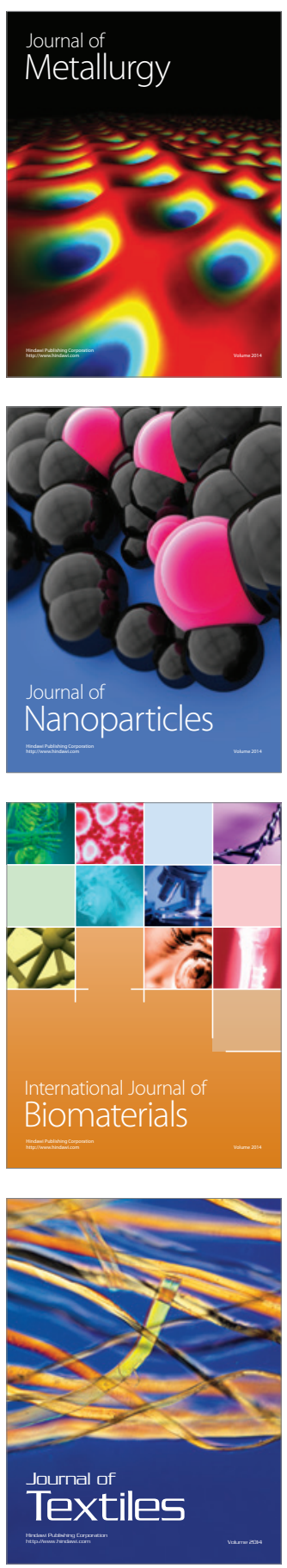